\title{
Average Atomic Number and Electron Backscattering in Compounds
}

\author{
John Donovan ${ }^{1}$, Jonathan Fellowes ${ }^{2}$ and Benjamin McMorran ${ }^{3}$ \\ 1. University of Oregon, CAMCOR, Eugene, Oregon, USA. \\ 2. School of Earth and Environmental Sciences, Univ of Manchester, Manchester, UK. \\ 3. University of Oregon, Department of Physics, Eugene, Oregon, USA.
}

It is often assumed that some signals (e.g., backscatter, continuum) emitted from electron solid interactions can be extrapolated from pure element observations to compounds by means of the assumption of average atomic number, or Z-bar $(\bar{Z})$ as it is often commonly known referred to. Typically the average $Z$ of a compound is assumed to be the sum of the mass fractions of the atomic numbers in the composition as seen here:

$$
\bar{Z}_{\left(c_{i}\right)}=\sum_{i=1}^{n} c_{i} Z_{i}
$$

eq. 1

where $c_{i}$ is the conventional mass fraction of element $i$, and $Z_{i}$ is the atomic number of element $i$. Now due to the fact that $\mathrm{A} / \mathrm{Z}$ is approximately a constant over the periodic table, this assumption is valid up to a point. However, since the neutron mass is essentially irrelevant in microanalytical situations where the electron beam energy is significantly less than $1 \mathrm{MeV}$ [1], perhaps we should examine this assumption more carefully. For electron backscattering we propose the following model based on what we call $\mathrm{Z}$ fraction, or $Z_{i}$ :

$$
\bar{Z}_{\left(z_{i}\right)}=\sum_{i=1}^{n} z_{i} Z_{i}
$$

where the $\mathrm{Z}$ fraction, $z_{i}$ is:

$$
z_{i}=\frac{a_{i} Z_{i}}{\sum_{i=1}^{n} a_{i} Z_{i}}
$$

and $a_{i}$ is the atomic fraction. However, in order to account for screening of the nuclear charge by inner orbital electrons we modify equation 3 by raising the atomic number to an exponent as seen here:

$$
\bar{Z}_{\left(z_{i}^{(x)}\right)}=\sum_{i=1}^{n} z_{i}^{(x)} Z_{i}
$$

where $z_{i}^{(x)}$ is the modified $Z$ fraction as seen here:

$$
z_{i}^{(x)}=\frac{a_{i} Z_{i}^{x}}{\sum_{i=1}^{n} a_{i} Z_{i}^{x}}
$$

and $x$ is an exponent less than 1.0. In figure 1 we plot the calculated backscatter fraction from high precision Monte Carlo modeling in PENEPMA 2012 [2], for both pure elements and selected compounds, which include elements with a range of $\mathrm{A} / \mathrm{Z}$ ratios. The pure elements from atomic number 10 to atomic number 85 are plotted as a line, and the compounds are plotted as symbols, where we have assumed mass fractional averaging for the calculation of average $Z$ in the compounds in (a) and using the modified $\mathrm{Z}$ fraction with an $x$ exponent of 0.7 for (b).

These results are consistent with empirical measurements of absorbed and faraday cup current as seen in figure 2, where the pure elements are plotted as black symbols and selected compounds are plotted as red symbols for mass fraction average $\mathrm{Z}$ in (a), and modified $\mathrm{Z}$ fraction in (b). 
References:

[1] Donovan, J.J., Pingitore, N.E., Westphal, A., Microscopy \& Microanalysis 9 (2003), p. 202.

[2] Llovet, X., Salvat, F., Microsc. Microanal. 23 (2017), p. 634.

(a)

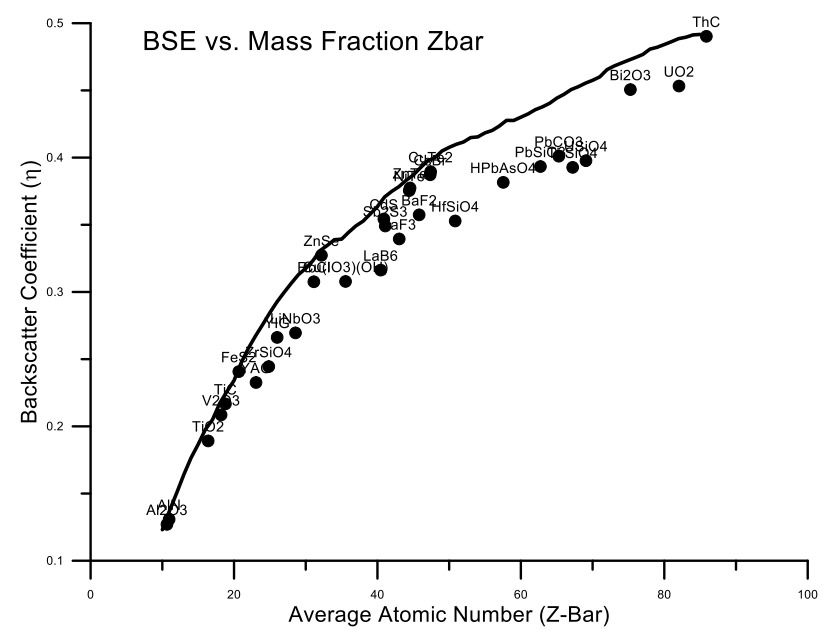

(b)

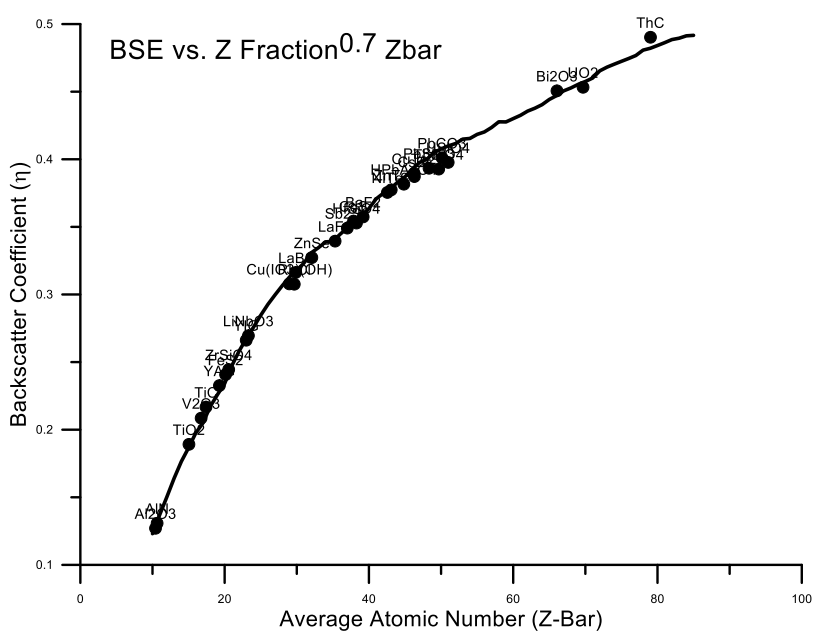

Figure 1. Monte Carlo calculations of electron backscatter fraction form pure elements plotted as a line, and compounds plotted as symbols, for mass fraction average $\mathrm{Z}$ in (a), and $\mathrm{Z}$ fraction average $\mathrm{Z}$ in (b).

(a)

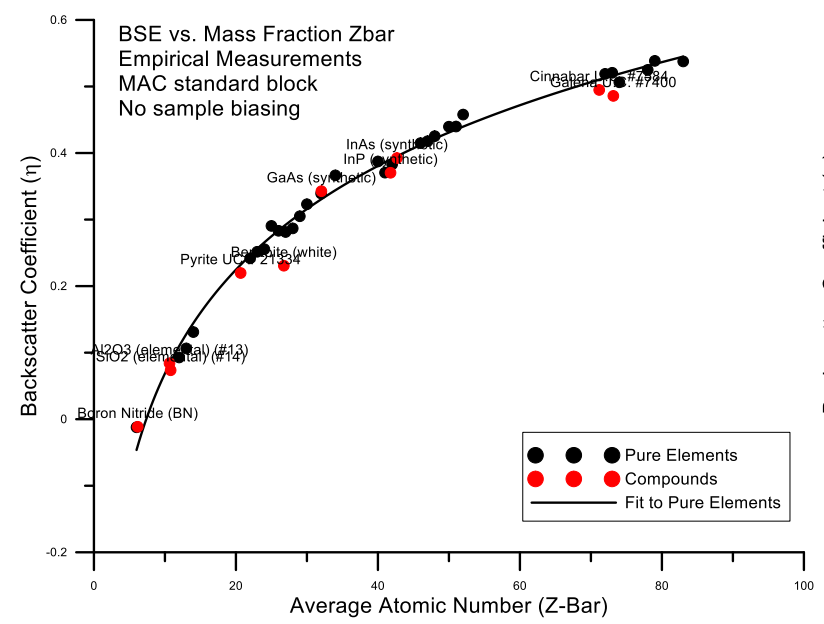

(b)

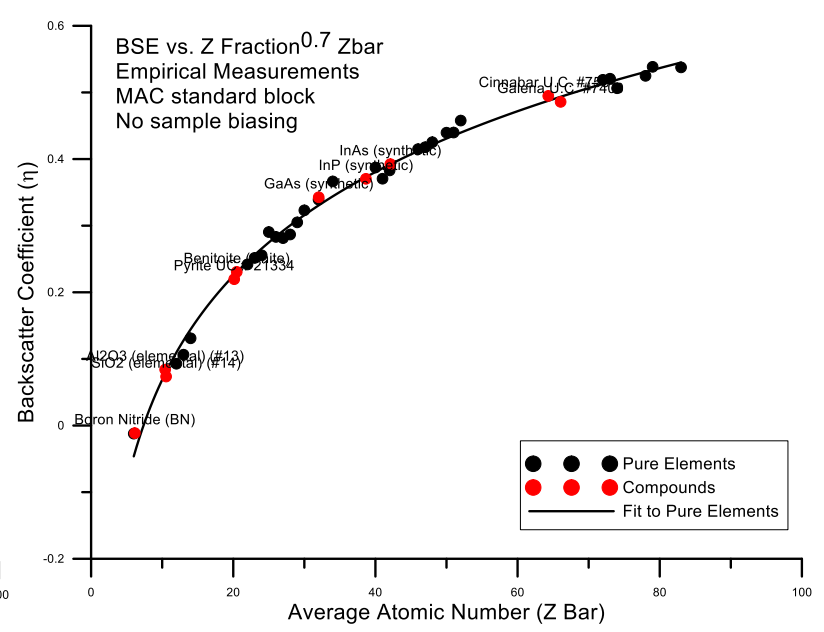

Figure 2. Measurements of electron backscatter on standard samples by measuring the ratio of the absorbed and faraday cup currents, i.e., $\eta=1-\frac{i_{\text {absorbed }}}{i_{\text {faraday }}}$. The backscatter fraction measured thusly is not accurate due to the re-absorption of secondary electrons, but for the purposes of a display of relative comparison it will suffice. Note Benitoite is a significantly different $\mathrm{A} / \mathrm{Z}$ ratio. 